\title{
Ratchet effect in a damped sine-Gordon system with additive and parametric ac driving forces
}

\author{
Elías Zamora-Sillero, ${ }^{1, *}$ Niurka R. Quintero, ${ }^{1, \dagger}$ and Franz G. Mertens ${ }^{2, \hbar}$ \\ ${ }^{1}$ Departamento de Física Aplicada I, E. U. P., Universidad de Sevilla, Virgen de África 7, 41011 Sevilla, Spain \\ ${ }^{2}$ Physikalisches Institut, Universität Bayreuth, Bayreuth, D-85440, Germany \\ (Received 2 June 2006; revised manuscript received 8 August 2006; published 13 October 2006)
}

\begin{abstract}
We study in detail the damped sine-Gordon equation, driven by two ac forces (one is added as a parametric perturbation and the other one in an additive way), as an example of soliton ratchets. By means of a collective coordinate approach we derive an analytical expression for the average velocity of the soliton, which allows us to show that this mechanism of transport requires certain relationships both between the frequencies and between the initial phases of the two ac forces. The control of the velocity by the damping coefficient and parameters of the ac forces is also presented and discussed. All these results are subsequently checked by means of simulations for the driven and damped sine-Gordon equation that we have studied.
\end{abstract}

DOI: 10.1103/PhysRevE.74.046607

PACS number(s): 05.45.Yv, 05.60.Cd

\section{INTRODUCTION}

The net transport of particles due to zero-average forces is known nowadays as ratchet mechanism or ratchet effect [1-3]. In the last decade, this phenomenon has attracted great interest after it was suggested as a working principle of molecular motors [4], where the energy of a chemical reaction can be used by certain molecules (myosin or actin) to move along periodic and asymmetric spatial structures (microtubules). The physics community notices that apparently a net current of particles appears when the spatial or/and temporal symmetries are broken in a proper way $[5,6]$ (for more details see [7] and a review [8]). Experimental realizations have shown that this phenomenon can also explain the motion of atoms in optical lattices [9], the net current of particles in granular materials [10], and the propagation of fluxons along Josephson Junctions (JJ) [11-14], to name only a few applications in other fields. This latter example corresponds to the motion of topological soliton, solution of the so-called sineGordon (sG) equation, where it has been possible to find out $[15,16]$ and also to explain [17-19] the basic mechanisms underlying the soliton ratchets in the overdamped and underdamped system as well, when the field potential is tilted. Other soliton ratchets have been found when the center of the kink moves in a ratchet potential (spatially asymmetric potential) $[12,20]$. Furthermore, it is known that the breaking of the symmetries can be also temporal instead of spatial, by using asymmetric time-periodic forces. Indeed, recently the rectification of the voltage due to a biharmonic asymmetric force in $\mathrm{JJ}$ has been found theoretically $[21,22]$ and confirmed experimentally [13]. In this problem a single harmonic is not enough for the appearance of the net transport; the direction of the soliton velocity can be managed by changing the phase of the driver [23], which is equivalent to changing the initial conditions. Therefore, the velocity averaged over initial conditions is zero. These results have also been confirmed by the experiments on JJ $[13,24]$.

\footnotetext{
*Electronic address: elias@euler.us.es

${ }^{\dagger}$ Electronic address: niurka@euler.us.es, niurka@us.es

${ }^{\ddagger}$ Electronic address: Franz.Mertens@uni-bayreuth.de
}

On the other hand, an ac force with a single harmonic added as a parametric excitation has also been used to deform the field potential (for the $\phi^{4}$ system see [25-27] and for the sine-Gordon chain see [18]). In particular, in [25,27] it has been found that the parametric ac force is able to act directly on the kink width causing its oscillatory motion, giving rise to the resonance phenomena. These results show the very different roles of the sole action of either an additive (acting directly on the momentum of the kink) or parametric single harmonic (acting on the kink width) of the ac force. The simultaneous action of these two forces has been analyzed first in [26] and later on in [18]. In the former work, drift motion of kinks in the $\phi^{4}$ model has been induced by the mixing of an additive and a parametric driver with the same frequency, where it has been found that the average velocity approaches its maximum value when the frequencies of the drivers approach the internal mode frequency of $\phi^{4}$. In the latter work, the motion of sG overdamped kinks in a deformable symmetric potential has been studied. For one kink in a chain the ratchet phenomenon has been explained using a pointlike particle representation with an oscillating mass. Contrary to the previous soliton ratchets, these two works deal with the net motion of the kinks in a deformable symmetric potential, either $\phi^{4}$ or sG. These models represent two examples of soliton ratchets. Motivated mainly by these works, we will study in detail the ratchet dynamics of the damped sine-Gordon kink under the action of parametric and additive ac forces.

In the present work we will extend the results obtained in $[18,26]$ in several directions. First, notice that, rather than the dynamics of the kink in a sG chain [18], we consider the continuum sine-Gordon model. By using a collective coordinate (CC) approach we establish a relationship both between the two frequencies and the two phases of the drivers for the occurrence and the disappearance of the net motion of the soliton, respectively. Indeed, we obtain (i) that the net motion of the kink is possible not only when the frequency of the parametric ac force $\omega_{1}$ is equal to the frequency of the additive driver $\omega_{2}$, but also appears when $\omega_{1}=\omega_{2} / 2, \omega_{2} / 3, \ldots$; (ii) the dependence of the average velocity on the initial phases as a direct consequence of the fact that the ratchet effect does not depend on the initial conditions; (iii) the dependence of the average velocity on the damping coefficient and the fre- 
quencies of the ac forces. Second, as will be seen below, despite obtaining the equations of motions that satisfy the $\mathrm{CC}$ in the sG model, the same equations hold, but with different parameters, for the $\phi^{4}$ model. Consequently, this procedure also will allow us to compare our results to the findings in [26] for the $\phi^{4}$ system. In this regard the role of the internal mode of $\phi^{4}$ in the resonance phenomena related to the direct excitation of the width of the kink by a parametric force [27] can be used to optimize the transport. Note that in the case of $\phi^{4}$ the localized internal mode with frequency $\Omega_{I}=\sqrt{\frac{3}{2}}$ is below the lower phonon frequency $\omega_{p h}=\sqrt{2}$, so we can choose the frequencies of the driver such that mainly the internal mode is excited, and to a lesser degree the phonons.

In order to show the generality of our results, in this paper we analyze the ratchet dynamic of the kink in the following perturbed sine-Gordon equation:

$$
\begin{aligned}
\phi_{t t} & -\phi_{x x}+\sin (\phi)\left[1+\epsilon_{1} \sin \left(\omega_{1} t+\theta_{1}\right)\right] \\
& =-\beta \phi_{t}+\epsilon_{2} \sin \left(\omega_{2} t+\theta_{2}\right)+\epsilon_{3},
\end{aligned}
$$

where the subindixes denote the partial derivatives of the field with respect to space $x$ and time $t ; \epsilon_{1}, \omega_{1}, \theta_{1}$, and $\epsilon_{2}, \omega_{2}$, $\theta_{2}$ represent the amplitude, frequency, and the phase of the parametric and additive ac forces, respectively; $\epsilon_{3}$ is the dc force and $\beta$ is a damping coefficient. Considering the parametric ac force in this way the topological charge of the kink is always conserved. Another advantage of this model is that analytically it is more tractable than, for instance, the deformed potential introduced in [18]. We would like to remark that in the study of the ratchet effect it is necessary to consider in this system $\epsilon_{3}=0$, i.e., the average of external forces should be zero. However, we keep this constant field with the aim of investigating whether the ac forces are able to reverse the motion of the kink induced by a dc external force.

The paper is organized as follows: In the next section we use a collective coordinate theory with two collective variables, namely the position $X(t)$ and the width $l(t)$ of the kink, with the aim of obtaining an approximate expression for the average velocity, $\langle\dot{X}\rangle \equiv\langle V\rangle$ (see the definition below). In Sec. III we check our analytical results by means of direct numerical simulations and we discuss the control of the soliton's transport by the external parameters of the periodic forces and the damping. Finally, in Sec. IV we summarize the main conclusions of the paper.

\section{AVERAGE VELOCITY FROM THE COLLECTIVE COORDINATE ANALYSIS}

The ratchet effect is characterized by a nonzero average velocity (or current) of particles and solitons under the actions of zero-average external forces. In the case of soliton ratchets, the $\mathrm{CC}$ theories have provided a useful tool not only to show the ratchet mechanisms but also to control the effect in each particular case $[17,19,21,22]$. In almost all these works, by means of the $\mathrm{CC}$ theories, approximate expressions for the average velocity have been obtained. In this section we use a CC theory in order to obtain the average velocity of the damped kink driven by two ac forces (a parametric and an additive one). Equation (1) can be rewritten as

$$
\begin{gathered}
\phi_{t t}-\phi_{x x}+\sin (\phi)=-\beta \phi_{t}+z(x, t, \phi), \\
z(x, t, \phi)=\epsilon\left[-a \sin \left(\omega_{1} t+\theta_{1}\right) \sin (\phi)+b \sin \left(\omega_{2} t+\theta_{2}\right)+c\right],
\end{gathered}
$$

where $a \equiv \epsilon_{1} / \epsilon, b \equiv \epsilon_{2} / \epsilon$, and $c \equiv \epsilon_{3} / \epsilon$ are rescaling factors, $\epsilon$ being a rescaling parameter. On the left-hand side (lhs) of Eq. (2), one identifies the unperturbed $\mathrm{sG}$ equation, whereas on the right-hand (rhs) $-\beta \phi_{t}$ and $z(x, t, \phi)$ represent the dissipation term and the external forces acting on the sG system, respectively. In the case of small amplitudes of the external forces and a small value of the damping coefficient, the terms on the rhs of Eq. (2) can be considered as perturbations of the sG system.

The additive ac force on the sG kink acts directly on its center of mass, and simultaneously it is able to excite the width of the kink via nonlinear coupling with the center of mass; whereas the parametric ac force only acts on the width $[25,27]$. Therefore, when these two forces act together on the kink, it is natural to consider in advance these two degrees of freedom and consequently we use the Rice Ansatz [4],

$$
\phi(x, t)=4 \operatorname{atan}\left[\exp \left(\frac{x-X(t)}{l(t)}\right)\right]
$$

as an approximate solution of (2) and (3). Using a Lagrangian formalism (for more details see [29]) one can find the evolution equations that satisfy the collective coordinates, the center $X(t)$, and the width $l(t)$ of the kink.

To this end we get rid of the dissipation term in Eq. (2) by using a change of variable in time $\tau=\exp (-\beta t)$ [29]. Then for the nondamped equation

$$
\phi_{\tau \tau}-\frac{\phi_{x x}}{\beta^{2} \tau^{2}}=-\frac{1}{\beta^{2} \tau^{2}} \frac{d U}{d \phi}+\frac{\widetilde{z}(x, \tau, \phi)}{\beta^{2} \tau^{2}},
$$

we can write the Lagrangian

$$
\begin{aligned}
L= & \int_{-\infty}^{+\infty} d x \mathcal{L}=\int_{-\infty}^{+\infty} d x\left\{\frac{1}{2} \phi_{\tau}^{2}-\frac{1}{2} \frac{\phi_{x}^{2}}{\beta^{2} \tau^{2}}\right. \\
& \left.-\frac{1}{\beta^{2} \tau^{2}}[U(\phi)+M(x, \tau, \phi)]\right\},
\end{aligned}
$$

where $\mathcal{L}$ is the Lagrangian density, $U(\phi)=1-\cos (\phi)$ the $\mathrm{s} \mathrm{G}$ potential, and $M(x, \tau, \phi)=-\int d \phi \widetilde{z}(x, \tau, \phi)$. The next step is to use the Rice Ansatz to obtain a Lagrangian as a function of the collective coordinates

$$
\begin{aligned}
L\left(X, X^{\prime}, l, l^{\prime}\right)= & \frac{M_{0} l_{0}}{2 l}\left(X^{\prime}\right)^{2}+\frac{\alpha M_{0} l_{0}}{2 l}\left(l^{\prime}\right)^{2}-\frac{M_{0}}{2 \beta^{2} \tau^{2}}\left(\frac{l_{0}}{l}+\frac{l}{l_{0}}\right) \\
& -\frac{1}{\beta^{2} \tau^{2}} \int_{-\infty}^{+\infty} l M(l \theta+X, \tau, \phi) d \theta
\end{aligned}
$$

where the prime denotes the derivative with respect to $\tau, \alpha$ $=\pi^{2} / 12, M_{0}=8$, and $l_{0}=1$ are the mass and the width of the kink at rest, respectively. By using the Lagrange equation we obtain the following evolution equations for the CCs: 


$$
\begin{aligned}
\frac{M_{0} l_{0}}{l(t)} \ddot{X}-\frac{M_{0} l_{0}}{l(t)^{2}} \dot{X} \dot{l}=-\beta \frac{M_{0} l_{0}}{l(t)} \dot{X}-\int_{-\infty}^{+\infty} z(X+y l, t, \phi) \phi_{y} d y, \\
\alpha\left[\dot{l}^{2}-2 \beta l \dot{l}-2 \ddot{l}\right]=\frac{l^{2}}{l_{0}^{2}}\left(\frac{l_{0}^{2}}{l^{2}} \dot{X}^{2}+1\right)-1 \\
+\frac{2 l^{2}}{M_{0} l_{0}} \int_{-\infty}^{+\infty} d y z(X+y l, t, \phi) y \phi_{y},
\end{aligned}
$$

where with dots we denote the derivative with respect to time $t$. Finally, substituting the perturbation (3) and the Ansatz (4) in Eqs. (8) and (9) and integrating, we obtain that $X(t)$ and $l(t)$, respectively, are governed by the following equations:

$$
\frac{M_{0} l_{0}}{l(t)} \ddot{X}-\frac{M_{0} l_{0}}{l(t)^{2}} \dot{X} \dot{l}=-\beta \frac{M_{0} l_{0}}{l(t)} \dot{X}-q \epsilon\left[b \sin \left(\omega_{2} t+\theta_{2}\right)+c\right]
$$

$$
\alpha\left[\dot{l}^{2}-2 \beta l \dot{l}-2 \ddot{l}\right]-\frac{l^{2}}{l_{0}^{2}}\left(\frac{l_{0}^{2}}{l^{2}} \dot{X}^{2}+1\right)+1-\frac{l^{2}}{l_{0}^{2}} \epsilon a \sin \left(\omega_{1} t+\theta_{1}\right)=0,
$$

where $q=2 \pi$ is the topological charge. For the $\phi^{4}$ system, i.e., if we replace the nonlinear term $\sin (\phi)$ in Eq. (1) by $-\phi+\phi^{3}$, the Rice Ansatz for the kink solution reads $\phi(x, t)$ $=\tanh [[x-X(t)] / l(t)]$, and after using the same CC theory we find that $X(t)$ and $l(t)$ also satisfy Eqs. (10) and (11), respectively, with $\alpha=\left(\pi^{2}-6\right) / 12, q=2, M_{0}=4 /(3 \sqrt{2})$, and $l_{0}=\sqrt{2}$. As a consequence, all equations derived in this section for the sine-Gordon will be also valid for $\phi^{4}$ (taking into account the appropriate values for the parameters according to the model). Note that after the change of variable

$$
\frac{d X}{d t}=\frac{P(t) l(t)}{M_{0} l_{0}}
$$

where $P(t) \equiv-\int_{-\infty}^{+\infty} d x \phi_{x} \phi_{t}$ represents the momentum of the system, Eq. (10) becomes in a linear equation for $P(t)$

$$
\frac{d P}{d t}=-\beta P(t)-q \epsilon\left[b \sin \left(\omega_{2} t+\theta_{2}\right)+c\right],
$$

and so it can be solved exactly. At this point we would like to remark that this equation for the momentum does not depend on the particular ansatz (4) but holds generally for the sineGordon model (for the proof follow the sketch developed in [30] for the sG equation under an additive ac force); in contrast to the $\phi^{4}$ model where it holds only in conjunction with the particular ansatz for the $\phi^{4}$ solitary wave.

The average velocity of the soliton center can be calculated straightforwardly from Eq. (12), obtaining

$$
\langle V\rangle \equiv\langle\dot{X}\rangle=\lim _{\tau \rightarrow+\infty} \frac{1}{\tau} \int_{0}^{\tau} d t \dot{X}(t) \equiv \lim _{\tau \rightarrow+\infty} \frac{1}{\tau} \int_{0}^{\tau} d t \frac{P(t) l(t)}{M_{0} l_{0}} .
$$

From this expression, note that the nonzero average velocity that characterizes the ratchet effect, appears only if, first, $P(t)$ and $l(t)$ oscillate with the same frequency (first resonance condition), and second, this effect appears unless the difference of phases between these two oscillatory functions satisfies certain relationships (second resonance condition).

The following analysis of the evolution equations (11)-(13) sheds light on the first resonance condition without solving these equations. First of all, note that the additive ac force acts directly on the momentum and therefore $P(t)$ oscillates with frequency $\omega_{2}$. Second, the parametric ac force acts directly on the width and as a consequence $l(t)$ oscillates with frequency $\omega_{1}$. Furthermore, the width of the kink is indirectly and parametrically driven with frequency $2 \omega_{2}$ through the term $\dot{X}^{2}(t) / l^{2}(t) \sim P^{2}(t)$, but this contribution to the spectrum of $l(t)$ does not cause any resonance with $P(t)$. On the other hand, from (11) we realize that since $l(t)$ satisfies the equation for a parametrically driven nonlinear oscillator, the higher harmonics of $\omega_{1}$ and $\omega_{2}$ will also be present in the spectrum of $l(t)$. Analogously, the higher harmonics of $\omega_{2}$ in $l(t)$ do not provide any resonance with $P(t)$, and hence the net transport is expected only for $\omega_{2}=\omega_{1}, \omega_{2}=2 \omega_{1}, \omega_{2}$ $=3 \omega_{1}$, etc. However, this is not sufficient to guarantee a nonzero average velocity. The second resonance condition imposes a certain relationship between the phases of these oscillatory functions (see the expressions for the average velocity below).

Let us now calculate the approximate expression for the average velocity. To begin with, we can solve Eq. (13), yielding

$$
\begin{gathered}
P(t)=A \exp (-\beta t)-\frac{q \epsilon c}{\beta}-\frac{q \epsilon b}{\sqrt{\beta^{2}+\omega_{2}^{2}}} \sin \left(\omega_{2} t+\theta_{2}+\psi\right), \\
\psi=-\operatorname{atan}\left(\frac{\omega_{2}}{\beta}\right), \quad A=P(0)+\frac{q \epsilon c}{\beta}+\frac{q \epsilon b}{\sqrt{\beta^{2}+\omega_{2}^{2}}} \sin \left(\theta_{2}+\psi\right),
\end{gathered}
$$

where $P(0)$ is the momentum at $t=0$. After a transient time $t \gg 1 / \beta$, the first term of (15) can be neglected, so the momentum reduces to

$$
P(t)=-\frac{q \epsilon c}{\beta}-\frac{q \epsilon b}{\sqrt{\beta^{2}+\omega_{2}^{2}}} \sin \left(\omega_{2} t+\theta_{2}+\psi\right),
$$

the superposition of a constant term and an oscillatory function, which oscillates with frequency $\omega_{2}$, as expected, regardless of the initial condition. Hence, $\dot{X}^{2} / l^{2} \sim P^{2}$ oscillates with frequencies $\omega_{2}$ (only if $c \neq 0$ ) and $2 \omega_{2}$. Now, we focus our attention on the second degree of freedom, the width of the kink. 
Assuming that after the transient time $l(t)$ oscillates around the unperturbed initial width $l_{0}$ of the static kink we expand this function in powers of $\epsilon$ around $l_{0}$,

$$
l(t)=l_{0}+\sum_{n=1}^{\infty} \epsilon^{n} l_{n}(t) .
$$

We can realize that in zero-order approximation, when $l(t)$ $\approx l_{0}$, the average velocity $\langle V\rangle$ is different from zero if and only if the dc field is present. Indeed, inserting the solution for $P(t)$ and taking $l(t) \approx l_{0}$ in Eq. (14) we obtain

$$
\langle V\rangle_{d c}=-\frac{q \epsilon_{3}}{M_{0} \beta},
$$

where we indicate with the subindex dc that this contribution comes from the dc field. At this point, we would like to stress that $\langle V\rangle_{d c}$ is not related at all to the ratchet effect since in this case the average of the external forces is not zero. However, we keep this constant field with the aim of investigating whether the ac forces are able to reverse the direction of the motion of the kink induced by a dc external force.

In order to find the average velocity related to the ratchet effect, we proceed as follows: First we insert (17) and (18) into (11) and obtain a hierarchy of equations in powers of $\epsilon$. Straightforward calculations show that in order $\epsilon, \epsilon^{2}$, and $\epsilon^{3}$, $l_{1}(t), l_{2}(t)$, and $l_{3}(t)$ satisfy the following linear differential equations:

$$
\begin{gathered}
\mathcal{L} l_{1}=-\frac{\Omega_{R}^{2} a l_{0}}{2} \sin \left(\omega_{1} t+\theta_{1}\right), \\
\mathcal{L} l_{2}=\frac{\Omega_{R}^{2} l_{1}^{2}}{2 l_{0}}-\frac{\Omega_{R}^{2} l_{1} a \sin \left(\omega_{1} t+\theta_{1}\right)}{2}+\frac{i_{1}^{2}}{2 l_{0}}-\frac{\Omega_{R}^{2} l_{0}}{2 M_{0}^{2}}\left(\frac{P}{\epsilon}\right)^{2} \\
\mathcal{L} l_{3}=-\frac{\Omega_{R}^{2}\left(l_{1}^{3}-2 l_{0} l_{1} l_{2}\right)}{2 l_{0}^{2}}-\frac{\Omega_{R}^{2} l_{2} a \sin \left(\omega_{1} t+\theta_{1}\right)}{2}-\frac{l_{1} i_{1}^{2}}{2 l_{0}^{2}}+\frac{i_{1} l_{2}}{l_{0}} \\
-\frac{\Omega_{R}^{2} l_{1}}{2 M_{0}^{2}}\left(\frac{P}{\epsilon}\right)^{2},
\end{gathered}
$$

respectively, where $\mathcal{L} \equiv d^{2} / d t^{2}+\beta d / d t+\Omega_{R}^{2}$ is a second-order linear differential operator, and $\Omega_{R}=1 / \sqrt{\alpha l_{0}}$ is the so-called Rice frequency [28]. The solutions of Eqs. (20) and (21), after the transient time is over, are given by

$$
\begin{gathered}
l_{1}(t)=a l_{10} \cos \left(\omega_{1} t+\theta_{1}+\delta\right), \\
l_{10} \equiv \frac{l_{0} \Omega_{R}^{2}}{2 \sqrt{\left(\omega_{1}^{2}-\Omega_{R}^{2}\right)^{2}+\omega_{1}^{2} \beta^{2}}}, \\
\delta=-\operatorname{atan}\left(\frac{\omega_{1}^{2}-\Omega_{R}^{2}}{\omega_{1} \beta}\right), \\
l_{2}(t)=l_{20}+a^{2} l_{21} \sin \left(2 \omega_{1} t+2 \theta_{1}+\delta+\chi+\tilde{\theta}_{1}\right) \\
+l_{22} \sin \left(2 \omega_{2} t+2 \theta_{2}+2 \psi+\tilde{\theta}_{2}\right),
\end{gathered}
$$

$$
\begin{gathered}
l_{21}=\frac{l_{10} \sqrt{l_{0}^{2} \Omega_{R}^{4}+l_{10}^{2}\left(\Omega_{R}^{2}-\omega_{1}^{2}\right)^{2}+2 l_{10} l_{0} \Omega_{R}^{2}\left(\Omega_{R}^{2}-\omega_{1}^{2}\right) \sin (\delta)}}{4 l_{0} \sqrt{\left(\Omega_{R}^{2}-4 \omega_{1}^{2}\right)^{2}+4 \beta^{2} \omega_{1}^{2}}}, \\
\chi=\operatorname{atan}\left(\frac{\left(\omega_{1}^{2}-\Omega_{R}^{2}\right)^{2}+2 \omega_{1}^{2} \beta^{2}}{\omega_{1} \beta\left(\Omega_{R}^{2}-\omega_{1}^{2}\right)}\right), \\
\tilde{\theta}_{n}=-\operatorname{atan}\left(\frac{4 \omega_{n}^{2}-\Omega_{R}^{2}}{2 \omega_{n} \beta}\right), \quad n=1,2 ;
\end{gathered}
$$

where we omit the expressions for the coefficients $l_{20}$ and $l_{22}$ for the sake of brevity. From these two approximations of the kink width we can observe that $l_{1}(t)$ oscillates with frequency $\omega_{1}$ and $l_{2}(t)$ with frequencies $2 \omega_{1}$ and $2 \omega_{2}$.

Substituting (17), (23), and (24) into (14), after integrating, we obtain

$$
\begin{aligned}
\langle V\rangle= & \langle V\rangle_{d c}-\frac{\epsilon_{1} \epsilon_{2} q \Omega_{R}^{2}}{4 M_{0} \sqrt{\beta^{2}+\omega^{2}} \sqrt{\left(\omega^{2}-\Omega_{R}^{2}\right)^{2}+\omega^{2} \beta^{2}}} \\
& \times \sin \left(\theta_{2}-\theta_{1}+\psi-\delta\right)+O\left(\epsilon^{3}\right),
\end{aligned}
$$

if $\omega_{1}=\omega_{2}=\omega$ (case I) and

$$
\begin{aligned}
\langle V\rangle= & \langle V\rangle_{d c}-\frac{q \epsilon_{1}^{2} \epsilon_{2} l_{21}}{2 M_{0} l_{0} \sqrt{\beta^{2}+\omega_{2}^{2}}} \cos \left(\theta_{2}-2 \theta_{1}+\psi-\delta-\chi-\tilde{\theta}_{1}\right) \\
& +O\left(\epsilon^{4}\right),
\end{aligned}
$$

if $\omega_{1}=\omega_{2} / 2$ (case II). These two expressions are the main results of this section.

Inserting (17), (23), and (24) on the rhs of Eq. (22) we find that in the spectrum of $l_{3}(t)$ should appear $\omega_{1}, 3 \omega_{1}$, and $2 \omega_{2} \pm \omega_{1}$. So, we expect that $l_{3}(t)$ resonates with $P(t)$ when $\omega_{1}=\omega_{2}$ (case I) or $\omega_{1}=\omega_{2} / 3$ (case III) and consequently a nonzero average velocity in $O\left(\epsilon^{4}\right)$ appears and can be calculated, but will give only a small contribution. Therefore, if the frequencies are equal (case I) this contribution will only marginally improve the formula (25), whereas if $\omega_{1}=\omega_{2} / 3$ (case III), it represents the first nonzero term appearing in $\langle V\rangle$ related to the ratchet effect.

\section{A. Zero-order correction for the average velocity. Reversion of the dc motion by ac forces}

From expressions (25) and (26) we observe that when the system is only driven by a constant field the velocity of the soliton is $\langle V\rangle_{d c} \sim-\epsilon_{3}$, so for positive (negative) values of $\epsilon_{3}$ the soliton moves to the left (right). This de movement could be compensated by the second terms in Eqs. (25) and (26) coming from the ac fields and then the net current can vanish. Furthermore, in some cases the direction of the movement of the kink due to the action of the constant force can be reversed by the ac forces (see Fig. 1).

\section{B. Ratchet effect}

After having analyzed the reversion of the dc motion by ac forces, we study the ratchet dynamic of the kink under the parametric and additive ac forces. So, hereafter $\epsilon_{3}=0$ and as a consequence $\langle V\rangle_{d c}=0$. 


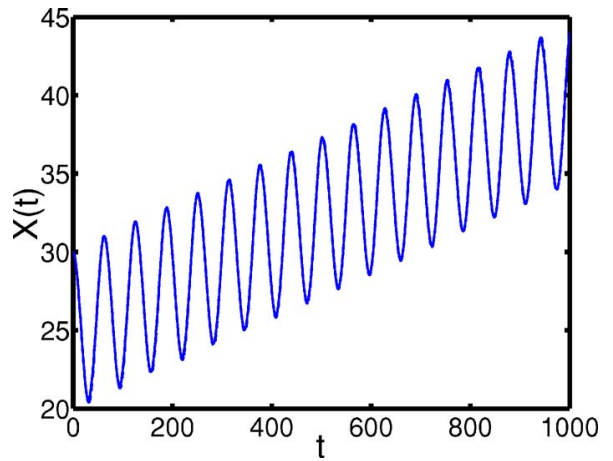

FIG. 1. (Color online) Reversion of the dc motion of the sG kink due to the parametric and additive ac forces. The evolution of the center of the kink computed from the simulations of the PDE (1) is represented by a solid line, whereas the dashed line stems from the numerical solution of the CC equations (11)-(13) (both curves are indistinguishable only to the eyes. The maximum of the absolute value of the difference between these two curves is around 0.4 for this interval of time). If the kink is driven only with a dc field with $\epsilon_{3}=0.001$ (see the text), it is expected that the kink moves to the left, but if in addition we consider also the parametric and additive ac forces with $\epsilon_{1}=0.2, \epsilon_{2}=0.1, \theta_{1}=0, \theta_{2}=0.8, \omega_{1}=\omega_{2}=0.1$, and $\beta$ $=0.1$ the kink moves in the opposite direction.

The CC approach and the perturbation theory applied up to the third-order correction predict a net transport of the sG soliton when the frequency $\omega_{2}$ of the additive ac force is $\omega_{2}=\omega_{1}, \omega_{2}=2 \omega_{1}, \omega_{2}=3 \omega_{1}$, being $\omega_{1}$ the frequency of the parametric ac force. From formulas (25) and (26), taking $\langle V\rangle_{d c}=0$, we note that the motion can be suppressed for certain values of the phases of these two forces, keeping constant the remaining parameters. These relationships between both the frequencies and the phases of the ac forces acting in the $\mathrm{sG}$ equation are necessary conditions to get and to suppress a net motion of the kink, respectively. We have shown that the nonzero average velocity appears at different orders of the perturbation theory in connection with these relationships. However, we have identified only one ratchet mechanism. Indeed, the momentum and the width of the kink resonate, oscillating with the same frequencies and appropriate phases, i.e., the additive ac force is locked to the oscillations of the width $[16,31]$.

The formulas (25) and (26) are also important because they allow us to control the ratchet velocity through the parameters of the ac forces and the damping. In the next section we check their validity by means of numerical simulations of the full nonlinear sG equation (1) and numerical solutions of the CC equations (11)-(13).

\section{NUMERICAL SOLUTIONS AND NUMERICAL SIMULATIONS}

In the previous section with the aim of characterizing the ratchet effect, we have used a CC approach that neglects the phonons. This implies that (i) the frequencies of the drivers $\omega_{1}$ and $\omega_{2}$ should be small compared to the lower frequency $\omega_{p h}=1$ of the phonons, (ii) the amplitudes of the ac forces shoud be small, i.e., $\epsilon_{1} \ll 1$ and $\epsilon_{2} \ll 1$, and (iii) increasing the damping coefficient the phonons can be avoided for the most part. In all our numerical simulations we will take into account all these constraints.

We have computed the numerical solutions of our CC equations (11)-(13) with the routine ode45 of Matlaboratory library [32] which uses a fourth-fifth-order Runge-Kutta method. We compute the solution of the Partial Differential Equation (PDE) (1) by a fourth-order Runge-Kutta scheme with space and time steps $\Delta x=0.1, \Delta t=0.01$, respectively, in the finite length domain $x \in[0,60]$ using as an initial condition a static sG kink at $x=30$.

In the following sections, we will present the numerical analysis in two parts according to the relationship between the frequencies of the ac forces we deal with.

\section{A. Resonance at equal frequencies $\omega_{2}=\omega_{1}=\omega$}

When the parametric and the additive ac forces act on the sG system, the ratchet dynamic of the kink is characterized by [in Eq. (25) take $\langle V\rangle_{d c}=0$ ]

$$
\begin{aligned}
\langle V\rangle= & -\frac{\epsilon_{1} \epsilon_{2} q \Omega_{R}^{2}}{4 M_{0} \sqrt{\beta^{2}+\omega^{2}} \sqrt{\left(\omega^{2}-\Omega_{R}^{2}\right)^{2}+\omega^{2} \beta^{2}}} \\
& \times \sin \left(\theta_{2}-\theta_{1}+\psi-\delta\right)+O\left(\epsilon^{3}\right) .
\end{aligned}
$$

From this expression the sinusoidal dependence of the average velocity on the phases can easily be seen, so there are some critical phases for which the motion vanishes (see Fig. 2). These critical phases can also be changed by the damping and the frequencies of the ac forces. Another relevant and interesting fact is that the motion can be controlled by the damping, as in the case of other soliton ratchets $[15,18,31]$. From (27) we note that the velocity decreases as a function of the damping; but since the phases $\psi$ and $\delta$ [see Eq. (16) and the expression for $\delta$ in (23)] are also functions of the damping, we expect that the average velocity does not decrease monotonically to zero as the damping is increased, but there is an optimal damping for the occurrence of net motion, or even the appearance of current reversal upon varying the damping [see [21,31]]. We show all these behaviors in Fig. 3.

Another interesting feature of this ratchet mechanism is related to the resonance behavior of the average velocity as a function of the frequency (see Fig. 4). We observe a maximum of $|\langle V\rangle|$ when $\omega=\Omega_{R} \approx 1$ as it is expected from Eq. (27). Note that we obtain a good agreement between the numerical solutions of the CCs and direct numerical simulations of the PDE only for low values of the frequency.

\section{B. Resonance at subharmonics of $\omega_{2}$}

In Sec. II we have shown that the ratchet motion can be observed also when $\omega_{1}=\omega_{2} / 2$ or $\omega_{1}=\omega_{2} / 3$. For the former frequency $\langle V\rangle$ is of order $\epsilon_{1}^{2} \epsilon_{2}$ and for the latter one $\langle V\rangle \neq 0$ only in the next-order approximation. A similar analysis of the average velocity can be carried out for the rest of the subharmonics of $\omega_{2}$. In particular, we have shown that when $\omega_{1}=\omega_{2} / 2$ the soliton moves with an average velocity given by [take in Eq. (26) $\langle V\rangle_{d c}=0$ ] 

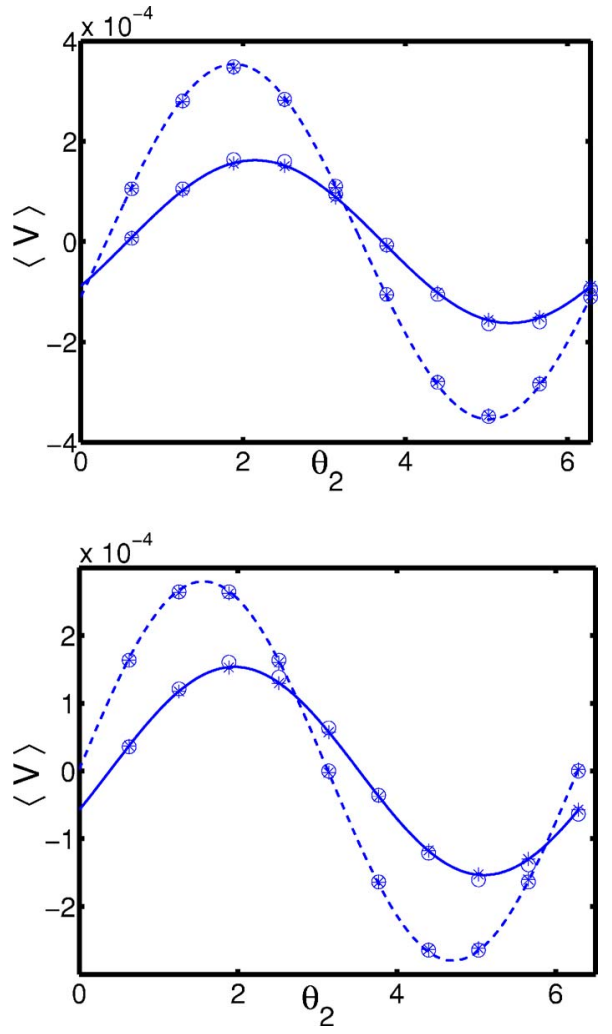

FIG. 2. (Color online) Average velocity vs the initial phase of the additive ac force for fixed $\theta_{1}=\pi / 4$. Both ac forces oscillate with the same frequency, either $\omega=0.1$ (dashed lines) or $\omega=0.25$ (solid lines). Other parameters of the simulations: $\epsilon_{1}=0.01, \epsilon_{2}=0.02$, and $\beta=0.05$ (top panel) and $\beta=0.1$ (bottom panel). Open circles: computed average velocity from the simulations of the PDE; stars: average velocity obtained from numerical solutions of the $\mathrm{CC}$ equations; solid and dashed lines: approximate average velocity (27).

$$
\langle V\rangle=-\frac{q \epsilon_{1}^{2} \epsilon_{2} l_{21}}{2 M_{0} l_{0} \sqrt{\beta^{2}+\omega_{2}^{2}}} \cos \left(\theta_{2}-2 \theta_{1}+\psi-\delta-\chi-\tilde{\theta}_{1}\right),
$$

where again we note a sinusoidal dependence on $\theta_{1}$ and $\theta_{2}$ (see Fig. 5), a polynomial dependence on the amplitudes of the ac forces (a direct consequence of perturbation theory), and a complex behavior of $\langle V\rangle$ as a function of the damping and frequencies. Indeed, the dissipation coefficient and frequencies not only can change the amplitude of the average velocity, but also change its sign (see Figs. 6 and 7). All these behaviors have been checked by means of numerical solutions of the CC equations (11)-(13) and simulations for the full PDE (1), where we have found an excellent agreement between our theory and numerical results only for small values of frequencies and amplitudes of the ac forces and not too small values of the damping coefficient, i.e., in cases where the phonons can be neglected (see Figs. 6 and $7)$. We would like to stress that $\langle V\rangle$ here must depend on $\theta_{2}-2 \theta_{1}$ as is shown in Eq. (28) and in Fig. 5. This behavior comes from the fact that the ratchet effect does not depend on the translations in time [33]. Another interesting remark, according to formulas (27) and (28), is that (in general) the
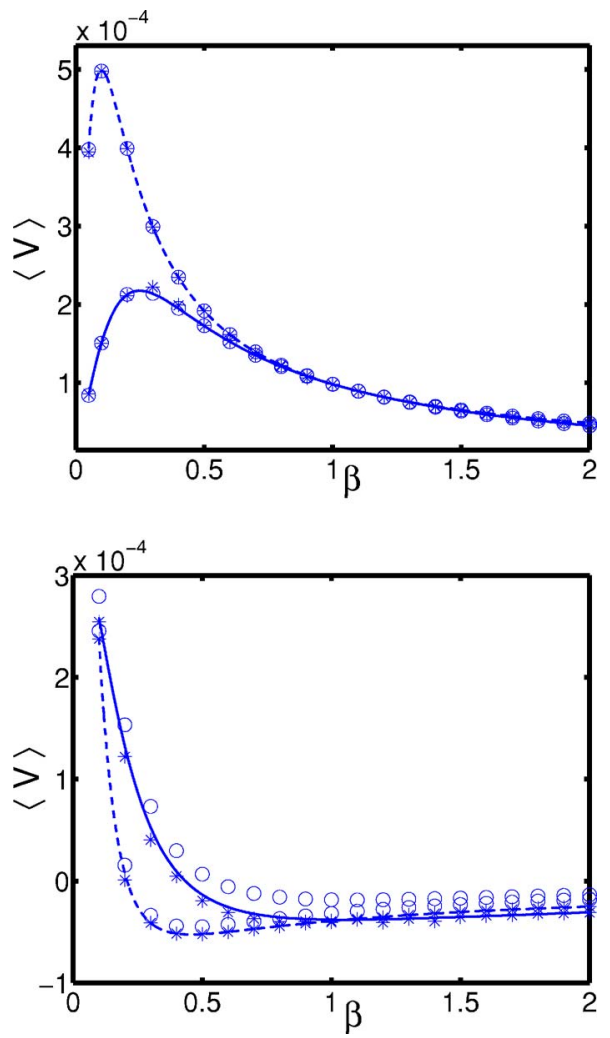

FIG. 3. (Color online) Average velocity vs the damping coefficient $\beta$ for $\epsilon_{1}=0.05, \epsilon_{2}=0.01, \theta_{1}=0$, and $\omega_{1}=\omega_{2}=\omega$. In the top panel, an optimal value of the damping is shown in both curves for $\theta_{2}=0, \omega=0.1$ (dashed line) and $\omega=0.25$ (solid line). In the bottom panel, a reversal current is observed for $\theta_{2}=2$ and $\omega=0.1$ (dashed line) and $\omega=0.25$ (solid line). Open circles and stars: $\langle V\rangle$ computed from the simulations of the PDE and from the numerical solutions of the CC equations, respectively. Solid and dashed lines: the approximate average velocity (27).

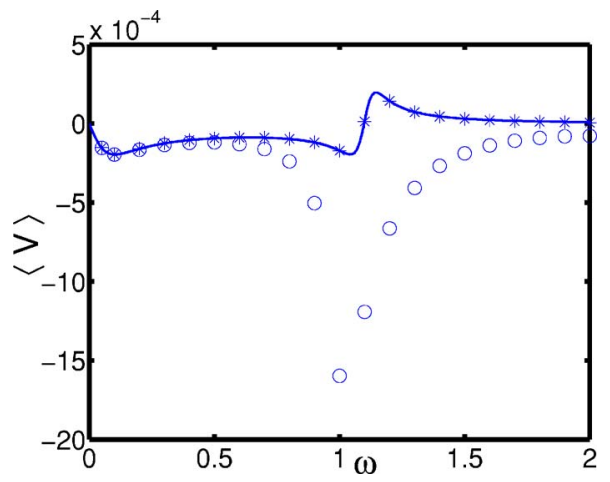

FIG. 4. (Color online) Resonance behavior of the kink ratchet velocity as a function of $\omega$. The parameters of the simulations are $\epsilon_{1}=0.01, \epsilon_{2}=0.02, \theta_{1}=\pi / 2, \theta_{2}=0$, and $\beta=0.1$. Open circles: $\langle V\rangle$ from the simulations of the PDE; stars: results obtained from numerical solutions of the $\mathrm{CC}$ equations; solid line: the average velocity (27). For higher values of the frequency, phonons are excited (the lowest phonon frequency for $\mathrm{sG}$ is 1 ), and so the collective coordinate theory with two degrees of freedom does not describe correctly the ratchet dynamic of the kink. 

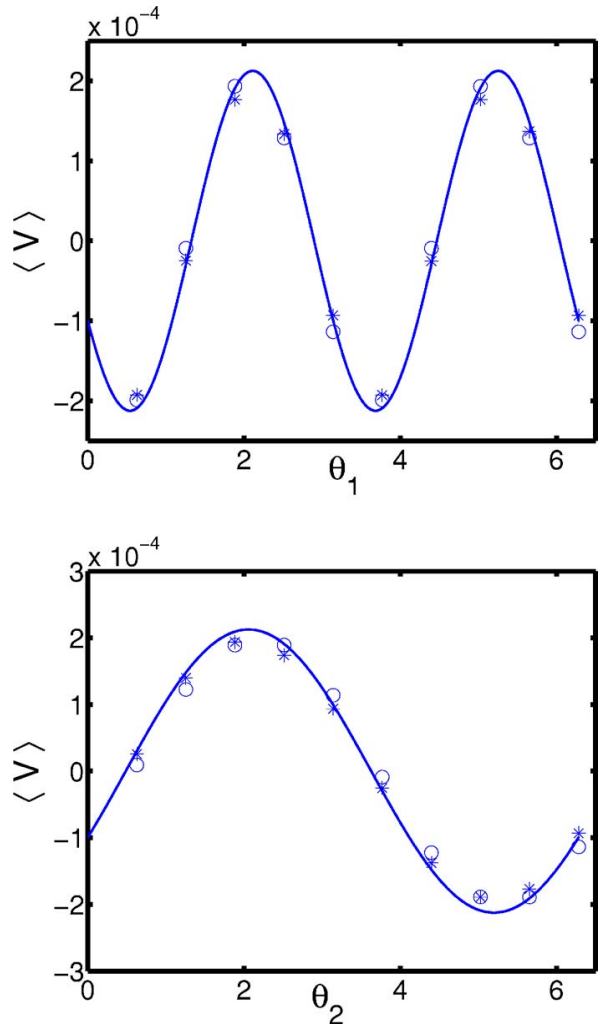

FIG. 5. (Color online) Average velocity as a function of the phases of the ac forces. Top panel: $\langle V\rangle$ as a function of the phase $\theta_{1}$ of the parametric ac force, when $\theta_{2}=0$. Bottom panel: $\langle V\rangle$ vs phase $\theta_{2}$ of the additive ac force, when $\theta_{1}=0$. Open circles and stars: $\langle V\rangle$ computed from the simulations of the PDE and from the numerical solutions of the $\mathrm{CC}$ equations, respectively. Solid lines: approximate average velocity (28). The parameters of the simulations are $\epsilon_{1}=0.1, \epsilon_{2}=0.1, \omega_{2}=2 \omega_{1}=0.2$, and $\beta=0.3$.

kink exhibits higher mobility if the frequencies of the ac forces are equal as was chosen in Refs. [18,26].

\section{CONCLUSIONS}

In this paper we have studied the dynamics of a damped sine-Gordon kink driven by parametric and additive ac forces. By using a CC approach with two collective variables, the center of mass of the kink and its width, we have obtained a system of Ordinary Differentail Equations (ODEs), from which we derived an approximate expression for the average velocity of the kink as a function of the system parameters [see Eqs. (27) and (28)]. We have confirmed numerically that the $\mathrm{CC}$ approach is valid when the excitation of phonons can be neglected. We have shown that the ratchet effect appears only if the momentum and the width of the soliton oscillate with the same frequency and with an appropriate difference of phases [34], however these conditions do not require necessarily equal frequencies of both drivers. Indeed, in first-order perturbation theory we have found that the net motion of the kink appears when the frequencies are equal $\omega_{1}=\omega_{2}$ (being $\omega_{1}$ and $\omega_{2}$ the frequency of the parametric and additive drivers, respectively), but in addition to this finding we have gone further, and in the
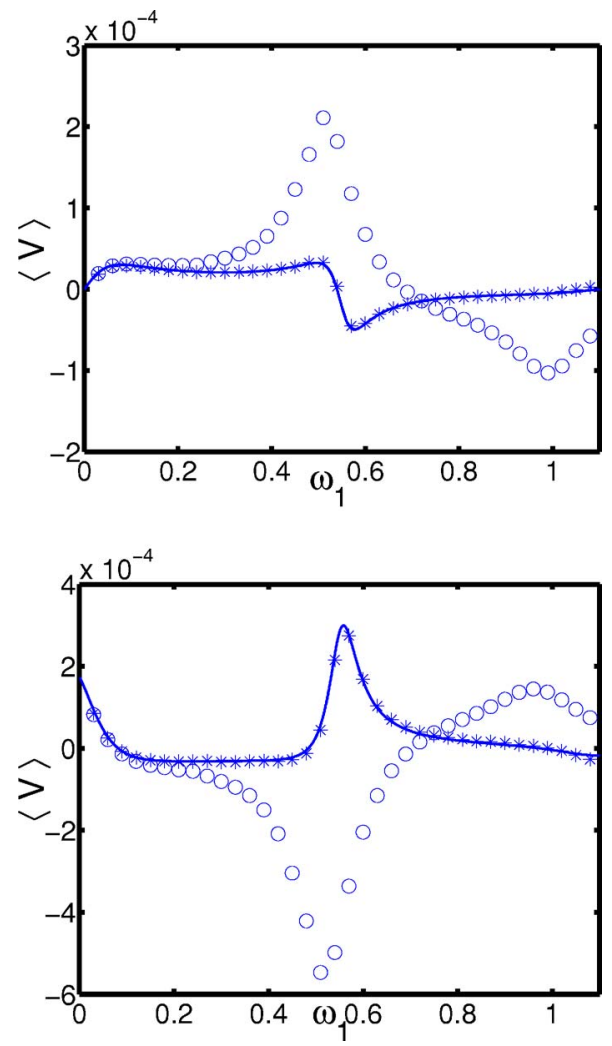

FIG. 6. (Color online) Average velocity vs $\omega_{1}=\omega_{2} / 2$. The other parameters of the numerical simulations are $\epsilon_{1}=\epsilon_{2}=0.05, \theta_{1}=0$, $\theta_{2}=\pi$, and $\beta=0.15$ (top panel) and $\epsilon_{1}=0.1$ and $\theta_{2}=5.5+\pi / 2$ (bottom panel). Open circles: simulations of PDE; stars: CC results; and solid lines: average velocity (28).

next-order approximations we find out that the net motion is also possible when $\omega_{1}=\omega_{2} / 2, \omega_{2} / 3, \ldots$, .

The main features of these kinds of soliton ratchets are the sinusoidal dependence of the average velocity $\langle V\rangle$ on the phases of both ac forces and the control of $\langle V\rangle$ by the damping and the frequencies of the forces. Varying these parameters it is not only possible to optimize $\langle V\rangle$, but also to reverse its direction, even in the presence of a constant dc force.

The role of the internal dynamics of the width of the kink in the ratchet mechanism, discussed in Sec. II, can be compared to the variable mass of the kink in a sG chain [18]. Indeed, when the frequencies of the drivers are equal, taking the adiabatic $(\omega \rightarrow 0)$ and overdamped $(\beta \gg 1)$ limits in Eq. $(27)$, we find that the average velocity

$$
\langle V\rangle=\frac{q l_{0}^{2} \epsilon_{1} \epsilon_{2}}{4 M_{0} \beta} \cos \left(\theta_{2}-\theta_{1}\right)
$$

is proportional to the amplitudes of each ac force and inverse proportional to the damping. In this limit we obtain practically the same results as obtained in [18] for a deformable potential (DP), where a similar phenomenon was studied for a string ratchet [see in [18] the Eq. 14 for DP]. Furthermore, taking into account in Eq. (10) $c=0$ and denoting $M(t)$ $=M_{0} l_{0} / l(t)$ we find that the center of the kink satisfies 

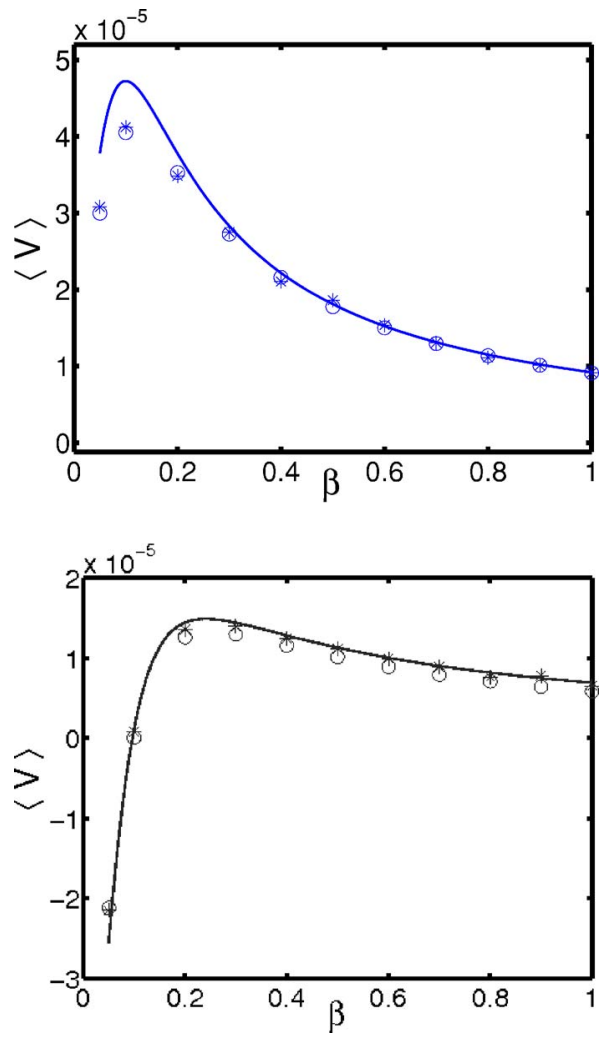

FIG. 7. (Color online) Average velocity vs the damping coefficient $\beta$ for $\omega_{1}=\omega_{2} / 2=0.05$. The other parameters of the numerical simulations are $\epsilon_{1}=\epsilon_{2}=0.05$ and $\theta_{1}=0$. In both panels, an optimal value of the damping is shown: in the top panel $\theta_{2}=\pi / 2$ and in the bottom panel, where a reversal current is observed, $\theta_{2}=\pi / 4$. Open circles and stars: $\langle V\rangle$ computed from the simulations of the PDE and from the numerical solutions of the $\mathrm{CC}$ equations, respectively. Solid lines: the approximate average velocity (28).

$$
M(t) \ddot{X}+\beta M(t) \dot{X}=-q \epsilon b \sin \left(\omega_{2} t+\theta_{2}\right)-\dot{M} \dot{X} .
$$

Comparing this expression with Eq. 13 obtained in [18], we realize that the inverse of the width of the kink considered by us is equivalent to the time-dependent mass introduced in [18]. From this equation it is also clear that the center of the kink moves in one direction because first it is driven by an additive ac force with an amplitude modulated by the width [31] and second the effective dissipation coefficient is timedependent function.
Contrary to the other soliton ratchets $[16,21,22]$, the soliton ratchet analyzed here corresponds to the case when the field potential in which the kink lies is modulated in time, but is still symmetric [18]. Furthermore, the kink is driven by a symmetric additive ac force. Concerning particle ratchet systems, the asymmetry, as has been pointed out recently in [35], is not needed to observe rectification. However, the ratchet mechanism is different. Indeed, when the particle is in a symmetric spatial potential modulated by a periodic force, the maximum value of the potential changes in time whereas the particle is driven also by an additive ac force. For the same frequencies of both forces it has been shown that the particle can "feel" the different heights of the potential barrier in both directions, giving rise to its net motion [35].

As a final remark, let us stress that the analysis on the soliton ratchet for the sine-Gordon kink carried out in this work can be extended also to other nonlinear systems supporting topological excitations. In particular, we have shown that for the underdamped $\phi^{4}$ equation driven by additive and parametric ac forces the same criteria for the appearance of the net motion of the kink should be fulfilled and consequently the ratchet effect manifests with the same mechanism and features. In this system the existence of the internal mode can be used to optimize the transport. Indeed, as pointed out in [26] [and also corroborated by our CC theory, see expression (27)], when the frequencies of both ac drivers are equal $\left(\omega_{1}=\omega_{2}=\omega\right)$ the average velocity of the domain wall can approach a maximum when $\omega$ is in the vicinity of the internal mode frequency $\Omega_{I} \approx \Omega_{R}$. In addition to this finding, we have shown that for other resonance cases, for instance, when $2 \omega_{1}=\omega_{2}$ or $3 \omega_{1}=\omega_{2}$, the maximum is expected at $\omega_{1}=\Omega_{R} / 2 \approx \Omega_{I} / 2$ or $\omega_{1}=\Omega_{R} / 3 \approx \Omega_{I} / 3$, respectively.

\section{ACKNOWLEDGMENTS}

We thank Fabio Marchesoni and Angel Sánchez for useful discussions on this work. We also acknowledge financial support by the Ministerio de Educación y Ciencia (MEC, Spain) and by DAAD (Germany) through "Acciones Integradas Hispano-Alemanas" Grant No. HA2004-0034-D/04/ 39957, and partially by a MEC Grant No. FIS2005-973 (E.Z.S., N.R.Q.), and by the Junta de Andalucía under Projects No. 00481 and No. FQM-0207 (E.Z.S., N.R.Q.). E.Z.S. was supported by the University of Seville.
[1] P. Hänggi and R. Bartussek, Nonlinear Physics of Complex System-Current Status and Future Trends, edited by J. Parisi et al., Lecture Notes in Physics Vol. 746 (Springer, Berlin, 1996), p. 476.

[2] R. D. Astumian and P. Hänggi, Phys. Today 55(11), 32 (2002).

[3] P. Hänggi, F. Marchesoni, and F. Nori, Ann. Phys. 14, 51 (2005)

[4] J. Maddox, Nature (London) 369, 181 (1994); M. O. Magnasco, Phys. Rev. Lett. 71, 1477 (1993); see also Ratchets and
Brownian Motors: Basics, Experiments and Applications, edited by H. Linke, Special issue of Material Sciences \& Processing 75, 167 (2002).

[5] A. Ajdari, D. Mukamel, L. Peliti, and J. Prost, J. Phys. I 4, 1551 (1994); S. Flach, O. Yevtushenko, and Y. Zolotaryuk, Phys. Rev. Lett. 84, 2358 (2000).

[6] S. Savel'ev, F. Marchesoni, P. Hänggi, and F. Nori, Europhys. Lett. 67, 179 (2004); Phys. Rev. E 70, 066109 (2004); M. Borromeo, P. Hänggi, and F. Marchesoni, J. Phys.: Condens. 
Matter 17, S3709 (2005).

[7] P. Reimann and P. Häggi, Appl. Phys. A 75, 169 (2002).

[8] P. Reimann, Phys. Rep. 361, 57 (2002).

[9] M. Schiavoni, L. Sánchez-Palencia, F. Renzoni, and G. Grynberg, Phys. Rev. Lett. 90, 094101 (2003); R. Gommers, S. Bergamini, and F. Renzoni, ibid. 95, 073003 (2005).

[10] Z. Farkas, P. Tegzes, A. Vukics, and T. Vicsek, Phys. Rev. E 60, 7022 (1999).

[11] I. Zapata, R. Bartussek, F. Sols, and P. Hänggi, Phys. Rev. Lett. 77, 2292 (1996).

[12] E. Goldobin, A. Sterck, and D. Koelle, Phys. Rev. E 63, 031111 (2001). For the experiments see M. Beck, E. Goldobin, M. Neuhaus, M. Siegel, R. Kleiner, and D. Koelle, Phys. Rev. Lett. 95, 090603 (2005).

[13] A. V. Ustinov, C. Coqui, A. Kemp, Y. Zolotaryuk, and M. Salerno, Phys. Rev. Lett. 93, 087001 (2004).

[14] F. Falo, P. J. Martínez, J. J. Mazo, and S. Cilla, Europhys. Lett. 45, 700 (1999); E. Trías, J. J. Mazo, F. Falo, and T. P. Orlando, Phys. Rev. E 61, 2257 (2000).

[15] F. Marchesoni, Phys. Rev. Lett. 77, 2364 (1996).

[16] Mario Salerno and Niurka R. Quintero, Phys. Rev. E 65, 025602(R) (2002).

[17] G. Costantini and F. Marchesoni, Phys. Rev. Lett. 87, 114102 (2001).

[18] G. Costantini, F. Marchesoni, and M. Borromeo, Phys. Rev. E 65, 051103 (2002).

[19] Niurka R. Quintero, Bernardo Sánchez-Rey, and Mario Salerno, Phys. Rev. E 72, 016610 (2005).

[20] L. Morales-Molina, F. G. Mertens, and A. Sánchez, Eur. Phys. J. B 37, 79 (2004); Phys. Rev. E 72, 016612 (2005).

[21] Mario Salerno and Yaroslav Zolotaryuk, Phys. Rev. E 65, 056603 (2002)
[22] Luis Morales-Molina, Niurka R. Quintero, Franz G. Mertens, and Angel Sánchez, Phys. Rev. Lett. 91, 234102 (2003).

[23] N. R. Quintero and A. Sánchez, Phys. Lett. A 247, 161 (1998); Eur. Phys. J. B 6, 133 (1998)

[24] E. Goldobin, B. A. Malomed, and A. V. Ustinov, Phys. Rev. E 65, 056613 (2002).

[25] Yuri S. Kivshar, Angel Sánchez, and Luis Vázquez, Phys. Rev. A 45, 1207 (1992); Yuri S. Kivshar, Niels Grønbech-Jensen, and Robert D. Parmentier, Phys. Rev. E 49, 4542 (1994).

[26] A. L. Sukstanskii and K. I. Primak, Phys. Rev. Lett. 75, 3029 (1995); Yu. S. Kivshar and A. Sánchez, ibid. 77, 582 (1996).

[27] Niurka R. Quintero, Angel Sánchez, and Franz G. Mertens, Phys. Rev. E 64, 046601 (2001).

[28] M. J. Rice and E. J. Mele, Solid State Commun. 35, 487 (1980).

[29] Niurka R. Quintero and Elías Zamora-Sillero, Physica D 197, 63 (2004); see also Sara Cuenda and Angel Sánchez, Chaos 15, 023502 (2005); Francisco J. Cao, Elías Zamora-Sillero, and Niurka R. Quintero, Phys. Rev. E 73, 056603 (2006).

[30] N. R. Quintero, Bernardo Sánchez-Rey, and Jesús CasadoPascual, Phys. Rev. E 71, 058601 (2005).

[31] L. Morales-Molina, Niurka R. Quintero, A. Sánchez, and F. G. Mertens, Chaos 16, 013117 (2006).

[32] J. R. Dormand and P. J. Prince, J. Comput. Appl. Math. 6, 19 (1980).

[33] Ricardo Chacón and Niurka R. Quintero (unpublished).

[34] The same mechanism is underlying the other soliton ratchets when the kink is in an asymmetric field potential $[18,19]$ or is driven by an asymmetric biharmonic force [21,22,31].

[35] M. Borromeo and F. Marchesoni, Chaos 15, 026110 (2005); Phys. Rev. E 73, 016142 (2006). 